\title{
ODOMETER ACTION ON RIESZ PRODUCT
}

\author{
MASAMICHI YOSHIDA
}

(Received 15 May 1993)

Communicated by A. H. Dooley

\begin{abstract}
We consider the Riesz product with a constant coefficient and odometer action over infinite product spaces. By studying the ratio set we can conclude the type of the above dynamical systems is III $_{1}$.
\end{abstract}

1991 Mathematics subject classification (Amer. Math. Soc.): 28D99.

\section{Introduction}

Brown and Dooley $[1,2]$ showed that the Riesz product (possibly with variable coefficients) is ergodic under the odometer action over an infinite product space. A well-known fact about Riesz products is that these measures are singular with respect to the Haar measure. In this paper we shall consider Riesz products with a constant coefficient $\alpha$ satisfying $-1<\alpha<1$. This kind of Riesz product gives a singular continuous probability. Furthermore let $\mathscr{F}_{n}$ be the sub- $\sigma$-algebra on the infinite product space generated by the projection onto the $n$-th coordinate. Then $\left\{\mathscr{F}_{n}\right\}$ is not independent with respect to Riesz products. So the behavior is different from the case of a product measure. Here we shall consider the following problem:

Determine the orbit equivalence type (see $[4,5]$ ) of the Riesz product with a constant coefficient under the odometer action.

For this purpose we must concentrate our attention to the behavior of the Jacobian of the Riesz product under odometer. Fortunately the Jacobian can be expressed explicitly. Then we shall compute its ratio set [5]. Finally we conclude that its type is $\mathrm{III}_{1}$.

(C) 1996 Australian Mathematical Society $0263-6115 / 96 \$ A 2.00+0.00$ 


\section{Type III 1 Riesz product measures}

Notation We shall denote by $X$ the infinite product space $\prod_{i=1}^{\infty}\{0,1\}$ and by $\Gamma$ the group of finite coordinate changes, that is,

$$
\Gamma=\left\{\gamma \in X: \exists N \geq 1 \text { such that } \forall k>N, \gamma_{k}=0\right\} .
$$

An element $\gamma \in \Gamma$ acts on $x \in X$ by

$$
(\gamma+x)_{k}=\gamma_{k}+x_{k}(\bmod 2) .
$$

For each $n \in \mathbb{N}$ we denote

$$
\Gamma^{n}=\left\{\gamma \in \Gamma: \gamma_{1}=\gamma_{2}=\cdots=\gamma_{n}=0\right\}
$$

and

$$
\Gamma_{n}=\left\{\gamma \in \Gamma: \forall k>n, \gamma_{k}=0\right\} .
$$

As usual, we shall equip $X$ with its Borel structure derived from the product topology. Riesz product measures over $X \quad$ Let $\alpha$ be $-1<\alpha<1$. For each $k \geq 1$ and $x \in X$ let

$$
g_{k}(x)=1+\alpha \cos 2 \pi\left(\frac{x_{k}}{2}+\frac{x_{k+1}}{2^{2}}+\frac{x_{k+2}}{2^{3}}+\cdots\right)
$$

and set $G_{k}(x)=\prod_{i=1}^{n} g_{i}(x)$. Then the measure $\mu$ (see [1]), defined by

$$
d \mu(x)=w^{*}-\lim _{n \rightarrow \infty} G_{n}(x) d \lambda(x)
$$

where $d \lambda(x)$ is the Bernoulli measure on $X$, is called the Riesz product corresponding to $\left\{G_{n}\right\}$. It is known $[1,2]$ that $\mu$ is $\Gamma$-quasi-invariant and ergodic, while the Jacobian $d \mu \gamma / d \mu(x)$, for each $\gamma \in \Gamma_{n}$, is $G_{n}(\gamma+x) / G_{n}(x)$. Furthermore, the family $\left\{G_{n}\right\}$ satisfies the following:

For every $\epsilon>0$, there exists $k \geq 1$ such that for every $x, y \in X$ and every $n \geq 1$,

$$
\rho(x, y)<\frac{1}{n+k} \Rightarrow\left|\frac{G_{n}(y)}{G_{n}(x)}-1\right|<\epsilon,
$$

where

$$
K(x, y)= \begin{cases}\min \left\{i \in \mathbb{N}: x_{i} \neq y_{i}\right\} & \text { if } x \neq y \\ \infty & \text { if } x=y\end{cases}
$$

and

$$
\rho(x, y)=K(x, y)^{-1} .
$$

This is called the property $\left(E_{1}\right)$ (see [1]).

The ratio set of $\Gamma$ For completeness we shall give a proof of the following theorem of Brown, Dooley and Lake [3]. 
THEOREM 1 (Brown, Dooley and Lake). Suppose that $\mu$ is a $\Gamma$-quasi-invariant ergodic probability on $X$. Let $r(\Gamma, \mu)$ be the ratio set of $\Gamma$.

Let $r \in \mathbb{R}_{+}$. Suppose that for every $\epsilon>0$ there exists $\beta>0$ such that for every $n \in \mathbb{N}$ and every $\gamma^{0} \in \Gamma_{n}$ there exists $\gamma \in \Gamma^{n}$ such that

$$
\mu\left\{x \in\left[\gamma^{0}\right]_{1}^{n}:\left|\frac{d \mu \gamma}{d \mu}(x)-r\right|<\epsilon\right\} / \mu\left[\gamma^{0}\right]_{1}^{n}>\beta
$$

where $\left[\gamma^{0}\right]_{1}^{n}=\left\{x \in X: x_{1}=\gamma_{1}^{0}, \ldots, x_{n}=\gamma_{n}^{0}\right\}$. Then $r \in r(\Gamma, \mu)$.

PROOF. We shall prove the case $r>0$. Let $0<\epsilon<r$. Choose $\beta$ according to the above assumption. Then for every $n \in \mathbb{N}$ and every $\gamma^{0} \in \Gamma_{n}$ there exists $\gamma \in \Gamma^{n}$ such that

$$
\mu\left[\gamma^{0}\right]_{1}^{n} \geq \mu\left(\gamma\left\{x \in\left[\gamma^{0}\right]_{n}^{1}:\left|\frac{d \mu \gamma}{d \mu}(x)-r\right|<\epsilon\right\}\right)>(r-\epsilon) \beta \mu\left[\gamma^{0}\right]_{1}^{n} .
$$

Hence $1>(r-\epsilon) \beta$. Let $A$ be an arbitary set of positive measure. By Lebesgue's density theorem, there exist $n \geq 1$ and $\gamma^{0} \in \Gamma_{n}$ such that

$$
\mu\left(A \cap\left[\gamma^{0}\right]_{1}^{n}\right)>\left(\left(1-\frac{\beta}{2}\right) \vee\left(1-\frac{\beta}{2}(r-\epsilon)\right)\right) \mu\left[\gamma^{0}\right]_{1}^{n} .
$$

On the other hand, we can choose $\gamma \in \Gamma^{n}$ such that $|d \mu \gamma / d \mu(x)-r|<\epsilon$ on a subset $B \subset\left[\gamma^{0}\right]_{1}^{n}$ of measure greater than $\beta \mu\left[\gamma^{0}\right]_{1}^{n}$. Set $C=A \cap B$. Note that $\gamma C \subset\left[\gamma^{0}\right]_{1}^{n}, \mu(C)>(\beta / 2) \mu\left[\gamma^{0}\right]_{1}^{n}$ and $|d \mu \gamma / d \mu(x)-r|<\epsilon(x \in C)$. It follows that $\mu(\gamma C)>(r-\epsilon)(\beta / 2) \mu\left[\gamma^{0}\right]_{1}^{n}$. Hence $\mu(A \cap \gamma C)>0$. We conclude that $r \in r(\Gamma, \mu)$.

Actually we will show that in the case of Riesz product measures with a constant coefficient, every positive number $r$ satisfies the condition of the above theorem.

The following lemma is used in [3].

LEMMA 1. Suppose that $\mu$ is the Riesz product measure with a constant coefficient $\alpha$ over $X$. Then for every $k \in \mathbb{N}$ there exists $\beta>0$ such that for every $n \in \mathbb{N}$ and $\gamma^{0} \in \Gamma_{n}$,

$$
\mu\left[\gamma^{0} 0^{k}\right]_{1}^{n+k} / \mu\left[\gamma^{0}\right]_{1}^{n}>\beta
$$

where $0^{k}$ is the word $00 \cdots 0$ (k-times).

PROOF. We first justify the following claim. 
(*) For every $k \in \mathbb{N}$ there exists $L>0$ such that for every $n \in \mathbb{N}, \gamma^{0} \in \Gamma_{n}$, $\gamma \in \Gamma^{n} \cap \Gamma_{n+k}$ and $x \in\left[\gamma^{0} 0^{k}\right]_{1}^{n+k}$,

$$
\frac{d \mu \gamma}{d \mu}(x)=\frac{G_{n+k}(\gamma+x)}{G_{n+k}(x)}<e^{L} .
$$

For each $k \in \mathbb{N}$ we set $L=2|\alpha| \pi /(1-|\alpha|)+k \log (1+|\alpha|) /(1-|\alpha|)$. Then, using the mean value theorem,

$$
\begin{aligned}
& \left|\log \frac{G_{n+k}(\gamma+x)}{G_{n+k}(x)}\right| \\
& \leq \sum_{i=1}^{n} \mid \log \left(1+\alpha \cos \pi\left(x_{i}+\frac{x_{i+1}}{2}+\cdots+\frac{x_{n}}{2^{n-i}}+\frac{x_{n+1}+\gamma_{n+1}}{2^{n+1-i}}+\cdots\right.\right. \\
& \left.\left.+\frac{x_{n+k}+\gamma_{n+k}}{2^{n+k-i}}+\frac{x_{n+k+1}}{2^{n+k+1-i}}+\cdots\right)\right) \\
& -\log \left(1+\alpha \cos \pi\left(x_{i}+\frac{x_{i+1}}{2}+\cdots+\frac{x_{n+1}}{2^{n+1-i}}+\cdots\right)\right) \mid+\sum_{i=n+1}^{n+k} \log \frac{1+|\alpha|}{1-|\alpha|} \\
& \leq \sum_{i=1}^{n} \frac{|\alpha| \pi}{1-|\alpha|}\left(\sum_{j=1}^{k} \frac{1}{2^{n+j-i}}\right)+k \log \frac{1+|\alpha|}{1-|\alpha|} \\
& <L \text {. }
\end{aligned}
$$

The claim (*) is proved. Then for every $\gamma \in \Gamma^{n} \cap \Gamma_{n+k}$,

$$
\mu\left[\gamma^{0} \gamma\right]_{1}^{n+k}=\int_{\left[\gamma^{0} 0^{k}\right]_{1}^{n+k}} \frac{d \mu \gamma}{d \mu}(x) d \mu(x)<e^{L} \mu\left[\gamma^{0} 0^{k}\right]_{1}^{n+k} .
$$

Set $\beta=2^{-k} e^{-L}$. Then $\beta$ has the required property.

Next set

$$
r_{1}=\prod_{i=0}^{\infty} \frac{1+\alpha \cos \left(2^{-j} \pi\right)}{1+\alpha}
$$

and, for each integer $K \geq 0$,

$$
r^{(K)}=\left(\prod_{j=0}^{K} \frac{1+\alpha \cos \left(2^{-j} \pi\right)}{1+\alpha}\right) \times\left(\prod_{i=0}^{\infty} \frac{1+\alpha \cos \left(2^{-j} \pi+2^{-(j+K+1)} \pi\right)}{1+\alpha}\right) .
$$

We will next show that the $r^{(K)}$ 's are distinct elements of the ratio set and have an accumulation point. Hence the Riesz product measure will be of type $\mathrm{III}_{1}$. 
LEMMA 2. $r_{1}, r^{(K)} \in r(\Gamma, \mu)(K \geq 0)$.

Before proceeding with the proof, we remark that by this lemma we can conclude that $(X, \mu, \Gamma)$ must be of type $\operatorname{III}_{\lambda}(0<\lambda<1)$, or $\mathrm{III}_{1}$.

PROOF. We shall prove $r_{1} \in r(\Gamma, \mu)$. Let $\epsilon>0$. Fix $\delta>0$ and an integer $m \geq 4$ such that $2 \delta+|\alpha| \pi / 2^{m-3}(1-|\alpha|)<\epsilon$. Take $k_{1} \in \mathbb{N}$ such that

$$
\left|\sum_{i=0}^{k} \log \left(\frac{1+\alpha \cos \left(\frac{\pi}{2^{i}}\right)}{1+\alpha}\right)-\log r_{1}\right|<\delta \quad\left(k \geq k_{1}\right) .
$$

By the property $\left(\mathrm{E}_{1}\right)$, there exists $k_{2} \in \mathbb{N}$ such that for every $n \in \mathbb{N}, \gamma \in \Gamma^{n+k_{2}}$ and $x \in X$,

$$
\left|\log \left(\frac{G_{n}(\gamma+x)}{G_{n}(x)}\right)\right|<\delta
$$

Set $k=\max \left\{k_{1}, k_{2}\right\}$.

Using Lemma 1, take $\beta>0$ such that

$$
\frac{\mu\left[\gamma^{0} 0^{k+m}\right]_{1}^{n+k+m}}{\mu\left[\gamma^{0}\right]_{1}^{n}}>\beta \quad\left(n \in \mathbb{N}, \gamma^{0} \in \Gamma_{n}\right) .
$$

Let $n \in \mathbb{N}$ and set $\gamma=\left(0^{n+k}, 1,0^{\infty}\right) \in \Gamma^{n+k} \cap \Gamma_{n+k+1}$. Then

$$
\frac{d \mu \gamma}{d \mu}(x)=\frac{G_{n}(\gamma+x)}{G_{n}(x)} \prod_{i=n+1}^{n+k+1} \frac{g_{i}(\gamma+x)}{g_{i}(x)} .
$$

We estimate:

$$
\begin{aligned}
\left|\log \frac{d \mu \gamma}{d \mu}(x)-\log r_{1}\right| \\
\leq\left|\log \frac{G_{n}(\gamma+x)}{G_{n}(x)}\right| \\
\quad+\mid \sum_{i=n+1}^{n+k+1} \log \left(\frac{1+\alpha \cos \pi\left(u_{i}+u_{i+1}+\cdots+u_{n+k}+\cdots+\tilde{u}_{n+k+1}+u_{n+k+2}+\cdots\right)}{1+\alpha \cos \pi\left(u_{i}+\cdots+u_{n+k+1}+\cdots\right)}\right) \\
\quad-\sum_{j=0}^{k} \log \left(\frac{1+\alpha \cos \frac{\pi}{2^{j}}}{1+\alpha}\right)|+| \sum_{j=0}^{k} \log \left(\frac{1+\alpha \cos \frac{\pi}{2^{j}}}{1+\alpha}\right)-\log r_{1} \mid \\
<2 \delta+\text { the second-term, }
\end{aligned}
$$

by setting $i=n+k+1-j, u_{s}=2^{-s+i} x_{s}, \tilde{u}_{s}=2^{-s+i}\left(x_{s}+1\right)$. 
Let $\gamma^{0} \in \Gamma_{n}$. Then for every $x \in\left[\gamma^{0} 0^{k+m}\right]_{1}^{n+k+m}$,

$$
\begin{aligned}
\left|\log \frac{d \mu \gamma}{d \mu}(x)-\log r_{1}\right| & <2 \delta+2 \sum_{i=n+1}^{n+k+1} \frac{|\alpha| \pi}{1-|\alpha|}\left(\sum_{j=1}^{\infty} \frac{x_{n+k+m+j}}{2^{n+k+m+j-i}}\right) \\
& \leq 2 \delta+2 \sum_{i=n+1}^{n+k+1} \frac{|\alpha| \pi}{1-|\alpha|} \frac{1}{2^{n+k+m-i}}<\epsilon .
\end{aligned}
$$

By (1),

$$
\mu\left\{x \in\left[\gamma^{0}\right]_{1}^{n}:\left|\log \frac{d \mu \gamma}{d \mu}(x)-\log r_{1}\right|<\epsilon\right\} / \mu\left[\gamma^{0}\right]_{1}^{n}>\beta .
$$

Then Theorem 1 asserts $r_{1} \in r(\Gamma, \mu)$.

In order to show $r^{(K)} \in r(\Gamma, \mu)$, it is enough to consider $\gamma^{(K)}=\left(0^{n+k}, 1,0^{K}, 1,0^{\infty}\right)$ as in the above argument.

We notice that $r^{(K)} \rightarrow r_{1}^{2}$ as $K \rightarrow \infty$.

\section{Conclusion}

THEOREM 2. The Riesz product with a constant coefficient is of type $\mathrm{III}_{1}$.

PROOF. By the remark after Lemma 2, $(X, \mu, \Gamma)$ must be of type III $_{1}$ or III $_{\lambda}$. We have claimed that $r^{(K)}, r_{1} \in r(\Gamma, \mu)$ and $r^{(K)} \rightarrow r_{1}^{2}$ as $K \rightarrow \infty$. Assume that there exists $0<\lambda<1$ such that the Riesz product is of type $\mathrm{III}_{\lambda}$. Then there exists $K_{0} \geq 0$ such that for every $K \geq K_{0}, r^{(K)}=r_{1}^{2}$. That is, for every $K \geq K_{0}$,

$$
1=\frac{r^{(K+1)}}{r^{(K)}}=\frac{1+\alpha \cos 2^{-(K+1)} \pi}{1+\alpha} \prod_{i=0}^{\infty} \frac{1+\alpha \cos \left(2^{-i} \pi+2^{-(i+K+2)} \pi\right)}{1+\alpha \cos \left(2^{-i} \pi+2^{-(i+K+1)} \pi\right)} .
$$

Therefore

$$
\frac{1+\alpha \cos 2^{-(K+1)} \pi}{1+\alpha}=\prod_{i=0}^{\infty} \frac{1+\alpha \cos \left(2^{-i} \pi+2^{-(i+K+1)} \pi\right)}{1+\alpha \cos \left(2^{-i} \pi+2^{-(i+K+2)} \pi\right)} \equiv P .
$$

On the other hand for every $0<\theta<\frac{1}{2}+\frac{1}{2^{K}}$,

$$
0<\delta \equiv \frac{\sin \pi\left(\frac{1}{2}+\frac{1}{2^{k}}\right)}{\pi\left(\frac{1}{2}+\frac{1}{2^{k}}\right)}<\frac{\sin \pi \theta}{\pi \theta}<1 .
$$

By the mean value theorem, there exist $\theta_{0 . K}$ and $\theta_{i, K}$ such that $1+1 / 2^{K+2}<\theta_{0, K}<$ $1+1 / 2^{K+1}, 1 / 2^{i}+1 / 2^{i+K+2}<\theta_{i, K}<1 / 2^{i}+1 / 2^{i+K+1}$ and

$$
\log P=-\alpha \pi \frac{1}{2^{K+2}} \frac{\sin \pi \theta_{0, K}}{1+\alpha \cos \pi \theta_{0, K}}+\sum_{i=1}^{\infty}\left(-\alpha \pi \frac{1}{2^{i+K+2}} \frac{\sin \pi \theta_{i, K}}{1+\alpha \cos \pi \theta_{i, K}}\right) .
$$


We notice that

$$
-\left(\frac{\pi}{1-\alpha}\right) \frac{1}{2^{K+1}}<\frac{\sin \pi \theta_{0, K}}{1+\alpha \cos \pi \theta_{0, K}}<-\left(\frac{\delta \pi}{1+\alpha}\right) \frac{1}{2^{K+2}}
$$

and that for every $i \geq 1$,

$$
\frac{\delta \pi}{1+\alpha}\left(\frac{1}{2^{i}}+\frac{1}{2^{i+K+2}}\right)<\frac{\sin \pi \theta_{i, K}}{1+\alpha \cos \pi \theta_{i, K}}<\frac{\pi}{1-\alpha}\left(\frac{1}{2^{i}}+\frac{1}{2^{i+K+1}}\right) .
$$

Then $\log P=\mathbf{O}\left(1 / 2^{K}\right)$ (as $\left.K \rightarrow \infty\right)$. On the other hand

$$
\log \frac{1+\alpha \cos \left(\pi / 2^{K+1}\right)}{1+\alpha}=\mathbf{O}\left(\frac{1}{2^{2 K}}\right) \quad(\text { as } K \rightarrow \infty) .
$$

This is a contradiction.

\section{Acknowledgement}

The author would like to express his sincere gratitude to Professor Anthony Dooley for extending warm hospitality during the time of the author's visit to the University of New South Wales and taking his time to discuss various aspects of the problem treated in this paper. And he is also grateful to Professor Yuji Ito for his encouragement and discussion.

\section{References}

[1] G. Brown and A. H. Dooley, 'Odometer actions and G-measures', Ergodic Theory Dynamical Systems 11 (1991), 279-307.

[2] G. Brown, 'Riesz products and generalized characters', Proc. London Math. Soc. 30 (1975), 209238.

[3] G. Brown, A. H. Dooley and J. Lake, 'On the Krieger-Araki-Woods ratio set', preprint.

[4] T. Hamachi, Y. Oka and M. Osikawa, 'A classification of ergodic nonsingular transformation groups', Mem. Fac. Sci. Kyushu Univ. Ser. A 18 (1974), 113-133.

[5] — 'Flows associated with ergodic nonsingular transformation groups', Publ. Res. Inst. Math. Sci. 11 (1975), 31-50.

\section{Department of Mathematics}

Osaka City University

Sugimoto, Sumiyoshi-ku

Osaka

Japan 